\title{
Neurobehavioral Aspects of Different Forms of Multiple Sclerosis
}

Nadia Ben Ali, Nouria Oudia, Saloua Mrabet ${ }^{\star}$, Mariem Kchaou, Slim Echebbi, Saloua Fray and Samir Belal

Department of Neurology, Charles Nicolle Hospital, Tunis, Tunisia

*Corresponding author: Saloua Mrabet, Department of Neurology, Charles Nicolle Hospital, Tunis, Tunisia, Tel: +216 97112 062; E-mail: salwamrabet85@hotmail.fr Received date: January 14, 2015; Accepted date: May 13, 2015; Published date: May 20, 2015

Copyright: @2015 Ali NB. This is an open-access article distributed under the terms of the Creative Commons Attribution License, which permits unrestricted use, distribution, and reproduction in any medium, provided the original author and source are credited.

\begin{abstract}
Introduction: Multiple sclerosis (MS) is an inflammatory disease of the central nervous system in young adults Cognitive impairment during MS has been overlooked for a long time and raises a renewed interest recently.
\end{abstract}

Objective: Determine prevalence of cognitive dysfunction in MS in a cohort hospital, particularities and factors affecting progression of cognitive dysfunction in individuals with MS.

Methods: Thirty patients (21 women and 9 men) with MS aged 18 to 43 years followed at the Neurology Department of Charles-Nicolle Hospital from 2008 to 2013 participated in this study. Patients participating in this study were chosen randomly without considering their cognitive status. Evaluation included a neuropsychological assessment, physical examination and brain and spine MRI.

Results: About $86 \%$ had cognitive impairment in at least one neuropsychological test. Predominant impaired domains were attention and information processing followed by verbal memory and executive functions. Depression was the most prevalent psychiatric disorder. Cognitive dysfunction was correlated with clinical form of MS, EDSS, radiological findings and disease's course.

Conclusion: Cognitive dysfunction might be more relevant to patients than mobility restrictions. Incorporating a systematic neuropsychological assessment in patients followed for MS allows psychosocial adaptation, monitoring disease activity and elucidating the effects of disease-modifying medications.

Keywords: Multiple sclerosis; Cognitive impairment; Tunisia

\section{Introduction}

Multiple sclerosis is an inflammatory disease of the central nervous system (CNS) in young adults with a presumed autoimmune origin. It affects more than 2.1 million people worldwide (National MS Society 2011) and is characterised by a heterogeneous broad spectrum and an unpredictable long-term outcome [1-2]. Cognitive impairment in MS has been underestimated. However, evidence emerging in the last 20 years suggests high prevalence rates of cognitive disturbances up to $70 \%$ when sensitive cognitive tests are applied. Attention, recent memory, information processing speed, executive functions, verbal intellectual ability, and visuospatial perception are the most involved [3-5].

Conflicting literature data report that cognitive impairment could be considered as an early feature of the disease or a consequence of its duration, course, and severity [6-8].Cognitive dysfunction has important clinical implications and might be more relevant to patients than mobility restrictions. Correlation between cognitive dysfunction and disease duration isn't clearly established. Recent studies have pointed out that it may affects patients at early stages of the disease. Cognitive impairment is detectable even in clinically isolated syndrome (CIS) and with increasing frequency in relapsing-remitting MS (RR-MS) and secondary progressive MS (SP-MS) [9]. Recent imaging and pathology studies have shown that MS affects white matter as well as grey matter. Unlike white matter lesion burden or distribution, grey matter atrophy has often been linked to cognitive impairment [10]. Nevertheless, relationship between cortical injury and diffuse white matter tracts damage and their respective contribution to cognitive dysfunction is still under investigations [11-12]. In this article we review cognitive profile in different forms of MS in a cohort hospital. Correlations with disability, clinical form, progression and imaging findings will be discussed.

\section{Patients and methods}

\section{Study design}

Thirty MS patients (21 women and 9 men) underwent the follow up study between 2008 to 2013. Patients were diagnosed with definite MS according to Macdonald criteria 2010 MS and gave informed consent before being included in the study. Physical disability was rated using Kurtzke's EDSS score. All patients participating in this study were chosen based on a clinical history of active MS and mild to moderate physical disability at disease's onset (EDSS 0-5.0), without considering their cognitive status. As a result, mean baseline neuropsychological test scores were within the normal range on all but one test (Word List Generation).Patients were in agreement with inclusion criteria such as age $>18$ years, follow up $>1$ year clinical. Exclusion criteria were no other neurological disease/head trauma, no known psychiatric illness, no alcohol/drug abuse and no medication which could influence cognition. 


\section{Demographic and clinical variables}

Demographic and clinical variables were recorded for each patient. Involvement of the CNS at onset was categorized according to initial symptoms: visual, sensory, motor, brainstem/cerebellar, bladder or unclassified impairment. Patients were grouped according to overall disease course: RR-MS, SP-MS and primary progressive MS (PP-MS).

\section{Neuropsychological assessment}

Neuropsychological functioning was assessed by tests covering: general cognitive functioning (similarities/picture completion from the Wechsler Adult Intelligence Scale (WAIS-III), psychomotor speed (the barrage test of Zazzo]), selective attention (the Stroop Color Naming Test [13]), working memory (the block tapping test (BTT), verbal learning and memory (the California Verbal Learning Test (CVLT) [12]), visuo-spatial memory (the Subtest WMS-R [14]) and executive function (Frontal Assessment Battery (FAB) [15]), semantic memory (fluency), global intelligence (Raven's Progressive Matrice), language (Ducarne's Subtest), praxia ( EMGD), visual gnosia (denomination) (Table 1).

\section{Cognitive functioning}

Global assessment of neuropsychological functioning based on the test results was made by a neuropsychologist. Some scores were graded as "no cognitive impairment present" and "cognitive impairment present". Criteria for "cognitive impairment" were a score of 1.5 standard deviations (SD) below the mean on at least one subtest in two of the four main functional areas (psychomotor speed, attention, learning/memory and executive functions).Deviations were based on norms, adjusted for age and educational level. Qualitative markers were consistent tendencies to perseverate or confabulate on learning and memory task, a clear impulsive response style with short performance time combined with many uncorrected false responses as well as inability to perform certain tasks.

All patients had a cerebral spinal MRI axial slices in sagittal and coronal T1, T2, FLAIR (Fluid attenuated inversion recovery), T2 * weighted images and gadolinium injection sequences.

\section{Statistical methods}

All statistical analyses were performed using SPSS for Windows version 15.0 (SPSS). We have calculated absolute frequencies and relative frequencies (percentages) for qualitative variables. We calculated averages, medians and standard deviations and determined extreme values for quantitative variables. Links between two quantitative variables were studied by the Pearson correlation coefficient. The Sig. (Significance) corresponds to the "p" if $<0.05$, then the " $r$ " is significantly different from zero: therefore, as it is + or -, there is a positive correlation or a statistically significant negative correlation. In all statistical tests, significance level was set at 0.05 .

\section{Results}

\section{General data}

Demographic data are summarized on Table 2. Mean age was 34.73 years; there were 21 women and 9 men. 17 patients (56.67\%) had a relapsing-remitting (RR) disease course; 9 (30\%) a secondaryprogressive (SP) disease course and $4(13,33 \%)$ a primary progressive form. Twenty-four patients (48\%) received immunotherapy. Educational level was academic in 26 patients. Four patients were illiterate.

\section{Age of onset}

Age of onset was 29.26 years (range from $18-43$ ). Age of onset was earlier on the relapsing remitting form (mean of 25,88yeras) comparatively with progressive forms of MS (32, 88 years for SP-MS and 35, 5 for the PP-MS).

\section{Disability}

Mean EDSS of total patients was 3, 02 ranging from 0,5 to 8 . It varies according to the outcome type of MS: EDSS was 1, 64 for RRMS, 4, 77 for SP-MS and 4,875 for the progressive form of MS.

\section{Treatment}

Treatment was based on immunomodulators (Interferon) and immunosuppressants (azathioprine and Natalizumab).

\section{Neuropsychological profile of MS}

About $86 \%$ had cognitive impairment in at least one neuropsychological test. The mean number of impaired tests per patient in the total population was 2.47 (range from 0 to 6 with an ecart type of 1.613). Predominant impaired domains were attention and information processing (73\% abnormal), followed by verbal memory $(53,3 \%)$ and executive functioning (40\%) (Figure1). 23 patients have depression $(76,6 \%)$.

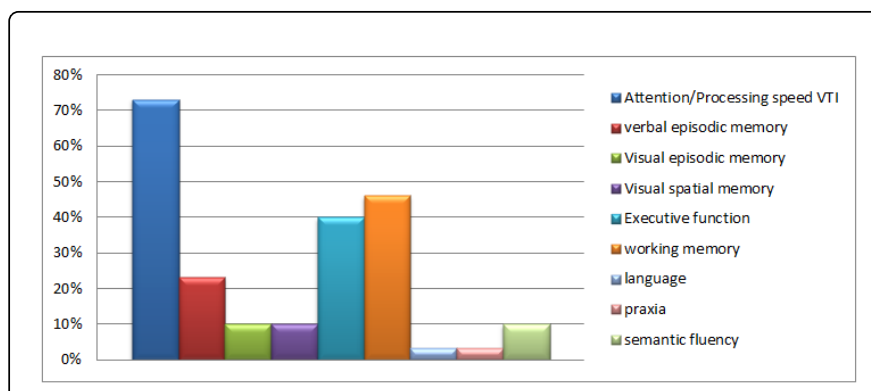

Figure 1: Neuropsychological profile of MS

\section{Attention}

21 Patients (70 \%) with MS had impairment on complex attention tasks. The pattern of performance has been interpreted as evidence of a reduced capacity to hold and manipulate information in mind, processes commonly conceptualized as functions of either working memory or supervisory attention.

\section{Memory}

Mnestic function was disrupted, with impaired memory reported in 10 to $53.3 \%$ of individuals with MS. Deficits in verbal memory may be more severe and appear earlier in the course of the disease; observed in $53.3 \%$ of patient with MS and followed by working memory impaired in 50\%. Visuo-spatial functions appear to be impaired in 16.5 $\%$ of patient with MS. Other aspects of memory, including semantic knowledge (impaired in $10 \%$ ), short-term memory, implicit learning and recognition, are generally preserved.

\section{Executive functions}


Page 3 of 6

Executive functions refer to such cognitive abilities as planning, problem-solving, and self-monitoring have frequently been found to be impaired in $40 \%$, conceptual reasoning appear to be a common feature of MS.

\section{Depression}

23 patients $(76,66 \%)$ had depression. Only 3 of them had a severe form (10\%). Mild depression seems to be more frequently, observed on $40 \%$.

\section{Correlates of cognitive dysfunction}

According to the type of MS, $100 \%$ of progressive form had cognitive impairment. The average number of tests impaired in the total population study was 2.47 (from 0 to 6) with an ecart type of 1,613 (Table 3). Otherwise, it differs if there is a progressive or remittent form of the disease with significant correlation if one variable is analysed (table 4, Figure 2). Depression was more frequent with progressive form of MS (88, $89 \%$ versus $64,7 \%$ on RRMS). A positive significant correlation was found between depression and severity of cognitive impairment (Figure 3). This average number varies according to the form of MS with statistically significant while between the progressive and the relapsing forms ((RR 1, 82; SP 3, 00; PP 4, 00) (Table 2).

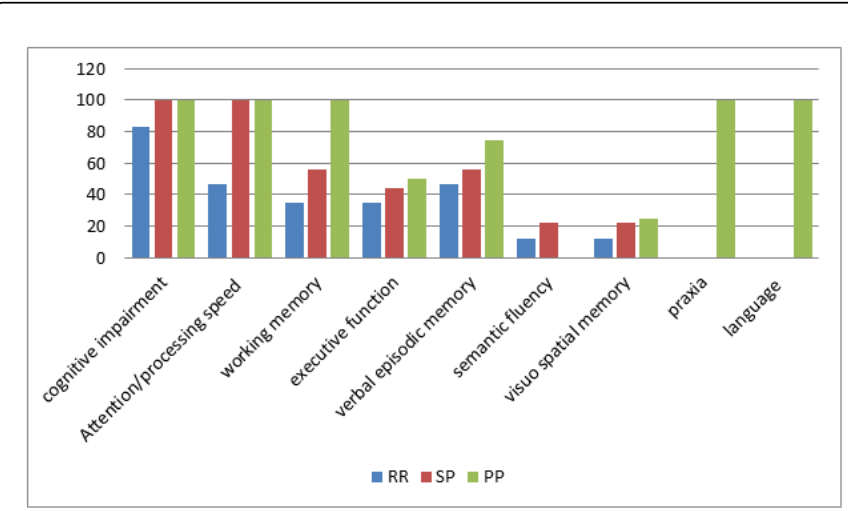

Figure 2: Cognitive impairment according to the form of MS

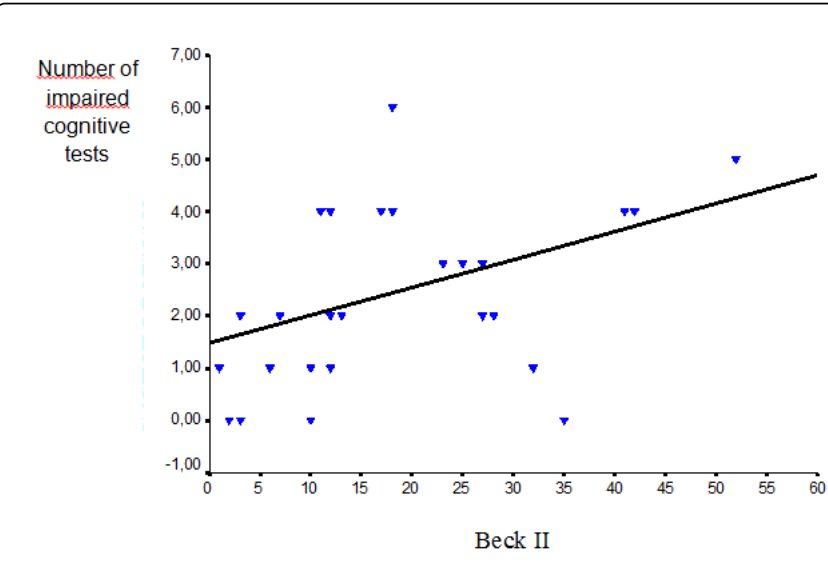

Figure 3: Correlation between depression and number of impaired tests
Correlations between any of the clinical data and cognitive function were observed. When investigating associations of markers that differ between cognitively impaired (CI) and cognitively preserved (CP) patients with cognitive function, a domain-specific pattern emerged. As expected from the lack of a group difference in disease duration, attention, the most frequent function impaired, significantly correlate with the duration, followed by visuo-spatial memory. Working memory correlates significantly with the form of $\mathrm{MS},(\mathrm{SP}=55,6 \%$; $\mathrm{PP}=100 \% ; \mathrm{RR}=35,3 \% ; \mathrm{p}=0,012$ ) but did not correlates with the EDSS or the disease duration (Table 6). Also, there is no correlation between verbal episodic memory and form of $\mathrm{MS}(\mathrm{RR}=47,1 \%$; $\mathrm{SP}=55,6 \%$; $\mathrm{PP}=75 \%) ; \mathrm{p}=0,594$, neither between EDSS and verbal memory $(\mathrm{p}=0$, $98)$ or disease duration and verbal memory $(\mathrm{p}=0,18)$ (Tables $5,7,8)$. While measures of episodic memory were more strongly associated with the progressive form, memory tasks (CVLT) not showed closer associations with different form of disease. Controversially, the number of impaired function is significantly correlated with the duration of disease (Figure 4). EDSS and cognition showed the strongest associations with cognitive performance (Figure 5). A significant positive correlation was found between EDSS and number of impaired tests $(\mathrm{r}=+0,470$ and $\mathrm{p}=0,009)$ (Table 9). Another significant correlation was found between depression found in $87 \%$ and number of impaired tests (Table 10).

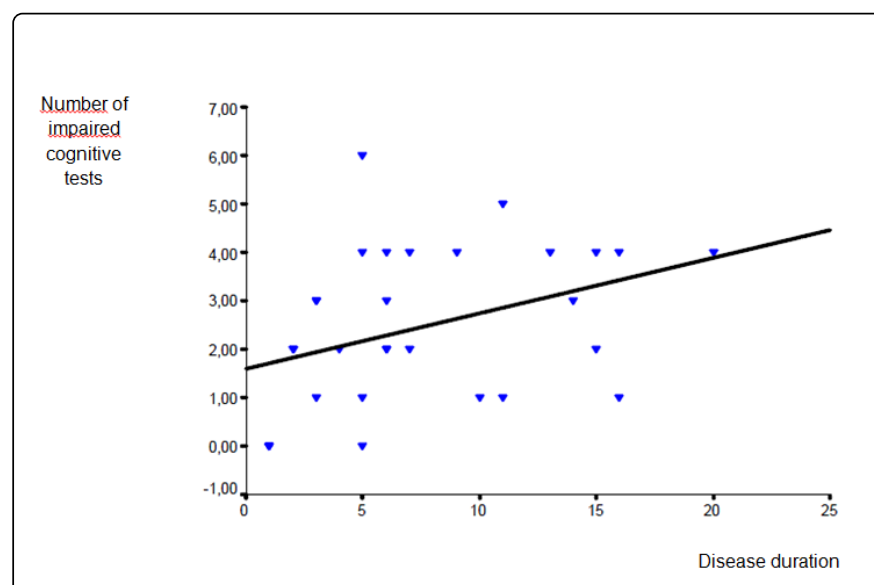

Figure 4: correlation between disease duration and cognitive impairment

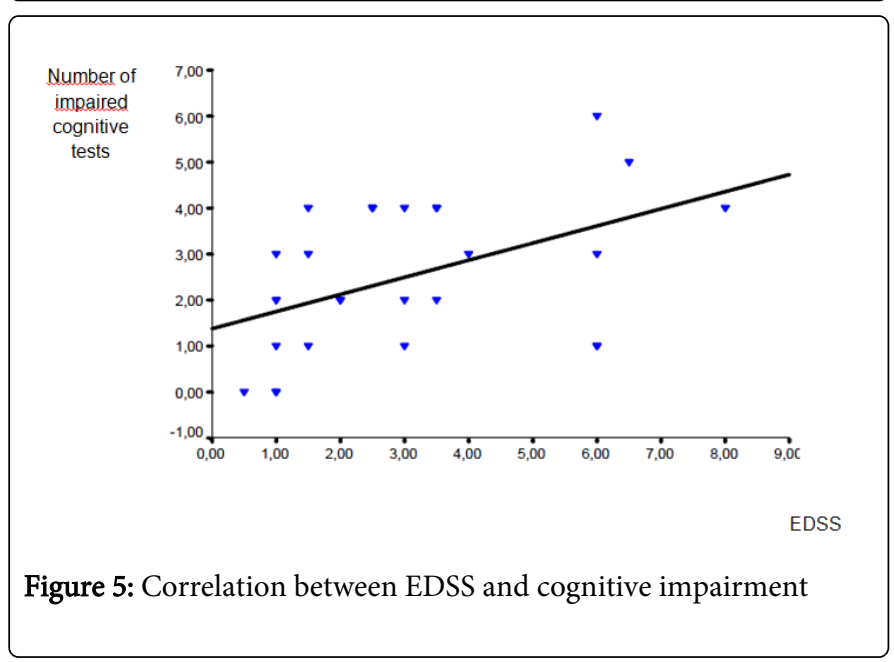




\section{Brain and Spine MRI findings}

Ten patients (33.3\%) had cortical atrophy and 8 patients $(26.7 \%)$ had atrophy of the callous cops. Average number of lesions was 22.5 (range 5 to 59). Lesions were located in: periventricular white matter (93.3\%), corpus callosum (83.3\%), semi oval center $(80 \%)$, cervical cord (53.3\%), cerebellar peduncles (53.3\%), internal capsule (46.7\%), protuberance, dorsolumbar cord (33.3\%) and bulb (26.7\%).

In our study, there is a significant correlation between cortical atrophy, corpus callosum atrophy and cognitive decline (respectively: $\mathrm{p}<0.05$ and $\mathrm{p}=0.049)$. Moreover, number of lesions was significantly correlated with cognitive impairment $(\mathrm{p}=0.022)$.

\section{Discussion}

Jean-Martin Charcot, the 19th-century neurologist who named multiple sclerosis, described cognitive and neuropsychiatric symptoms during this condition [19]. As neurologists stressed the white matter nature of multiple sclerosis, they further downplayed any cognitive or other "gray matter problems."

During the past two decades, there has been an increasing awareness of cognitive disturbances in MS which occur in about 35 to $70 \%$ of patients [1-5].

Cognitive impairment in early-onset MS has also been reported and has a major impact on social functioning $[6,16]$. It's a main cause of reduced work hours or unemployment in patients with MS [17].

In the world literature, data is based on studies from Western Europe, North America and Latin America. To our Knowledge, there are no available studies from other regions of the world, particularly from North Africa or the Maghreb.

Neurobehavioral aspects of MS include both cognitive disturbances and neuropsychiatric disorders. Cognitive impairment has been identified at all stages of the disease: clinically isolated syndrome (CIS), relapsing remitting multiple sclerosis (RRMS), secondary progressive multiple sclerosis (SPMS) and primary progressive multiple sclerosis (PPMS). Cognition can also predict future disease progression and can be a risk factor of further physical disability [18].

Cognitive difficulties in MS involve memory retrieval, mental processing speed, reasoning and goal-oriented behavior, verbal fluency, and visuo-spatial skills. Neuropsychiatric disturbances are primarily mood disorders. Cognitive impairment occurs in 40-65\% MS [19]. In our cohort, cognitive impairment was found in about $86 \%$ in at least one neuropsychological test. Cognitive deficits are most pronounced in secondary progressive disease, such as our population where $100 \%$ of progressive form had impairment.

Controversially, some data showed that cognitive profile did not correlate with physical disability. Karlińska I et al. concluded that correlation between physical disability and cognitive impairment was not significant, except for memory deficits and psychomotor speed [20].

Others demonstrate that cognitive deficits are more frequent and pronounced in chronic progressive MS and tend to worsen over time. Significant impairment occurs in almost all studied cognitive domains such as episodic memory, executive function, and processing speed [21]. For most cases, severity of cognitive impairment significantly correlates with physical disability and with depression severity $[15,22,23]$.
In our study, EDSS showed the strongest associations with cognitive performance.

Moreover, it has been suggested that slowing velocity of information processing and, secondarily, deficient non speeded central executive skills may be core to the cognitive deficits characteristic of multiple sclerosis patients $[15,24]$. Memory is the most affected cognitive deficits in multiple sclerosis; impaired memory is reported in $40-60 \%$ of individuals and about 10 to $53.3 \%$ in our study $[25,26]$. Furthermore, beginning during the early stages of the disease, memory impairments may be prominent [27].

Impairment appears to be characterized by deficits in retrieval from long-term storage. Free recall of verbal as well as visuo-spatial material appears to be impaired in MS [28]. Although, deficit in verbal memory may be more severe and appears earlier in the course of the disease. In contrast to secondary memory, primary memory, which is responsible for immediate recall, is generally intact. Multiple sclerosis patients perform comparable to normal on implicit memory tasks such as motor skill acquisition and priming. Primary problem in MS in memory functioning is in acquisition of new information. Verbal memory deficits is more associated with a progressive disease course, however, visuospatial memory deficits is more reported in RR MS. It has been suggested that automatic memory processing is intact in multiple sclerosis, but impairment in memory, in meta memory, and in other cognitive tasks becomes evident over time when patients rely on conscious processes [29].

Fatigue is a weakening symptom of MS ranging from 53 to $90 \%$ [30]. Degree of fatigue is related to processing speed impairment. Cognitive slowing has been observed to be significantly associated with the type of MS: secondary progressive forms show the most extensive range of deficits, closely followed by primary progressive forms; relapsing-remitting forms appear to be much less affected [31]. Our results also coincide with longitudinal studies showing that cognitive slowing reached the attention was found on $47.1 \%$ in our RR-MS group compared to $100 \%$ of patients with a progressive form. This significant difference $(p=0.003)$ was reported in several studies [19].

As previously mentioned, our results show a rate of executive dysfunction about $40 \%$. Working strategies seem to be the most frequent subtype of impaired executive dysfunction. According to Cerezo Garcia $\mathrm{M}$ et al., cognitive flexibility, inhibition and abstraction ability were the most deficient components of executive functions [23]. A positive correlation was found between motor disability and severity of executive functions in our study and in the international literature: patients with the worst performance were those with progressive forms and a high EDSS. Given the complexity of executive functions, further studies are needed to clarify degree and types of executive difficulties experienced by those with MS and relation between executive dysfunction and other types of cognitive impairment.

Psychiatric disturbances are common in MS. Depression is frequent and is reported in up to $60 \%$ of people with MS in our study $(76,6 \%)$ as well as in literature. Moreover, it's more common than in other chronic diseases [32]. Fatigue, anxiety, apathy are also prominent symptoms in MS and aggravate cognitive functioning [33].

Sleep disorders and high levels of sexual dysfunction are also common in patients with MS but often overlooked.

Information processing efficiency refers to the ability to maintain and manipulate information in the brain for a short time period and to the speed with which one can process that information [32]. The first 
cognitive difficulty, in our study, refers to a processing speed. These data are consistent with world literature. Processing speed, visual learning and memory seem to be most commonly affected in MS [34].

Several studies, on this subject, have agreed that a close link is established between disease course and cognitive decline. When comparing subtypes of MS, patients with a PPMS or SPMS course have typically demonstrated a greater severity of cognitive impairment than patients diagnosed with a relapsing-remitting course. Furthermore, different subtypes of MS have also been associated with different cognitive profiles. PPMS and SPMS patients may be more likely than RRMS patients to suffer from attention, speed of processing, executive and abstraction deficits. Moreover, RRMS patients may be more likely than healthy controls to suffer from memory deficits. Relationship between cognitive impairment and MRI parameters is discussed in many studies. In our analysis and in other published data, cognitive impairment is correlated with brain atrophy and brain lesions' volume [5].

Atrophic frontal lobes and cognitive changes are frequently associated during MS. Gray matter demyelinating lesions (cerebral cortex, hippocampus, thalamus and basal ganglia) and white matter ones causing interconnectivity between structures highlighted are the major causes of neurobehavioral changes in MS [35]. MRI analysis seems to be insufficient. Studies based on functional neuroimaging are more reliable and needed.

\section{Conclusion}

Results of this study highlight ongoing need to perform more longitudinal studies of cognitive impairment in patients with relapsing and progressive forms of MS. Cognitive dysfunction in MS has a multi factorial etiology. It's a major source of vocational disability, social impairment, and impoverished quality of life during the course of MS.

Cognitive deficits may occur early in the course of the disease since first demyelinating episode that can precede clinically definite MS. Early cognitive dysfunction is a predicator of conversion to clinically definitive MS and possibly for further disabling evolution. As such, systematically assessment of cognitive function is strongly recommended in the first time examination. Regular monitoring of cognitive functioning is necessary.

\section{References}

1. Buchanan RJ, Minden SL, Chakravorty BJ, Hatcher W, Tyry T, et al. (2010) A pilot study of young adults with multiple sclerosis: demographic, disease, treatment, and psychosocial characteristics. Disabil Health J 3: 262-270.

2. Confavreux C, Compston A (2005) The natural history of multiple sclerosis. In: Compston A, McAlpine D, eds. Mc Alpine's Multiple Sclerosis, 4th edn. Philadelphia: Churchill Livingstone Elsevier 2005: 183-272.

3. Rao SM, Leo GJ, Bernardin L, Unverzagt F (1991) Cognitive dysfunction in multiple sclerosis. I. Frequency, patterns, and prediction. Neurology 41: 685-691.

4. Patti F, Amato MP, Trojano M, Bastianello S, Tola MR, Goretti B, et al. (2009) COGIMUS Study Group. Cognitive impairment and its relation with disease measures in mildly disabled patients with relapsingremitting multiple sclerosis: baseline results from the Cognitive Impairment in Multiple Sclerosis (COGIMUS) study. Mult Scler 15: 779-788.

5. Ochi H (2014) [Cognitive impairment in multiple sclerosis]. Brain Nerve 66: 1201-1209.
6. Koopmans RA, Li DK, Oger JJ, Kastrukoff LF, Jardine C, et al. (1989) Chronic progressive multiple sclerosis: serial magnetic resonance brain imaging over six months. Ann Neurol 26: 248-256.

7. Comi G, Filippi M, Martinelli V, Sirabian G, Visciani A, et al. (1993) Brain magnetic resonance imaging correlates of cognitive impairment in multiple sclerosis. J Neurol Sci 115 Suppl: S66-73.

8. Kujala P, Portin R, Ruutiainen J (1997) The progress of cognitive decline in multiple sclerosis. A controlled 3-year follow-up. Brain $120: 289-297$.

9. Feuillet L, Reuter F, Audoin B, Malikova I, Barrau K, et al. (2007) Early cognitive impairment in patients with clinically isolated syndrome suggestive of multiple sclerosis. Mult Scler 13: 124-127.

10. Kawachi I, Saji E, Nishizawa M (2014) [Gray matter lesions and cognitive impairment in multiple sclerosis]. Rinsho Shinkeigaku 54: 1060-1062.

11. Rogers JM, Panegyres PK (2007) Cognitive impairment in multiple sclerosis: evidence-based analysis and recommendations. J Clin Neurosci 14: 919-927.

12. Sperling RA, Guttmann CR, Hohol MJ, Warfield SK, Jakab M, et al. (2001) Regional magnetic resonance imaging lesion burden and cognitive function in multiple sclerosis: a longitudinal study. Arch Neurol 58: $115-121$.

13. Lynch SG, Parmenter BA, Denney DR (2005) The association between cognitive impairment and physical disability in multiple sclerosis. Mult Scler 11: 469-476.

14. Charcot JM (1877) Lectures on the diseases of the nervous system. Lecture V. Translation by Sigerson B London: The New Sydenham Society.

15. Arnett PA (2005) Longitudinal consistency of the relationship between depression symptoms and cognitive functioning in multiple sclerosis. CNS Spectr 10: 372-382.

16. Wishart H, Sharpe D (1997) Neuropsychological aspects of multiple sclerosis: a quantitative review. J Clin Exp Neuropsychol 19: 810-824.

17. De Sonneville LM, Boringa JB, Reuling IE, Lazeron RH, Adèr HJ, et al. (2002) Information processing characteristics in subtypes of multiple sclerosis. Neuropsychologia 40: 1751-1765.

18. Portaccio E, Stromillo ML, Goretti B, Zipoli V, Siracusa G, et al. (2009) Neuropsychological and MRI measures predict short-term evolution in benign multiple sclerosis. Neurology 73: 498-503.

19. Jongen PJ, Ter Horst AT, Brands AM (2012) Cognitive impairment in multiple sclerosis. Minerva Med 103: 73-96.

20. KarliÅ,ska I, Siger M, Lewa ^,ska M, Selmaj K (2008) Cognitive impairment in patients with relapsing-remitting multiple sclerosis. The correlation with MRI lesion volume. Neurol Neurochir Pol 42: 416-423.

21. Langdon DW (2011) Cognition in multiple sclerosis. Curr Opin Neurol 24: 244-249.

22. Lynch SG, Parmenter BA, Denney DR (2005) The association between cognitive impairment and physical disability in multiple sclerosis. Mult Scler 11: 469-476.

23. Cerezo García M, Martín Plasencia P, Aladro Benito Y (2015) Alteration profile of executive functions in multiple sclerosis. Acta Neurol Scand 131: 313-320.

24. Arnett PA, Rao SM, Grafman J, Bernardin L, Luchetta T, et al. (1997) Executive functions in multiple sclerosis: an analysis of temporal ordering, semantic encoding, and planning abilities. Neuropsychology 11: 535-544.

25. Tsolaki M, Drevelegas A, Karachristianou S, Kapinas K, Divanoglou D, et al. (1994) Correlation of dementia, neuropsychological and MRI findings in multiple sclerosis. Dementia 5: 48-52.

26. Daly E, Komaroff AL, Bloomingdale K, Wilson S, Albert MS (2001) Neuropsychological function in patients with chronic fatigue syndrome, multiple sclerosis, and depression. Appl Neuropsychol 8: 12-22.

27. Zarei M, Chandran S, Compston A, Hodges J (2003) Cognitive presentation of multiple sclerosis: evidence for a cortical variant. J Neurol Neurosurg Psychiatry 74: 872-877.

28. Grafman J (1990) In: Rao SM, editor. Neurobehavioral aspects of multiple sclerosis. New York: Oxford University Press 102-117. 
Citation: Ali NB, Oudia Z, Mrabet S, Kchaou M, Echebbi S, et al. (2015) Neurobehavioral Aspects of Different Forms of Multiple Sclerosis . J Neurol Neurophysiol 6: 293. doi:10.4172/2155-9562.1000293

Page 6 of 6

29. Gaudino EA, Chiaravalloti ND, DeLuca J, Diamond BJ (2001) A comparison of memory performance in relapsing-remitting, primary progressive and secondary progressive, multiple sclerosis. Neuropsychiatry Neuropsychol Behav Neurol 14: 32-44.

30. Strober LB1 (2015) Fatigue in multiple sclerosis: a look at the role of poor sleep. Front Neurol 6: 21.

31. Kroencke DC, Lynch SG, Denney DR (2000) Fatigue in multiple sclerosis: relationship to depression, disability, and disease pattern. Mult Scler 6: 131-136.

32. Chiaravalloti ND, DeLuca J (2008) Cognitive impairment in multiple sclerosis. Lancet Neurol 7: 1139-1151.
33. Marrie RA, Reingold S, Cohen J, Stuve O, Trojano M, et al. (2015) The incidence and prevalence of psychiatric disorders in multiple sclerosis: A systematic review. Mult Scler 21: 305-317.

34. Benedict RH, Cookfair D, Gavett R, Gunther M, Munschauer F, et al. (2006) Validity of the minimal assessment of cognitive function in multiple sclerosis (MACFIMS). J Int Neuropsychol Soc 12: 549-558.

35. Jongen PJ, Ter Horst AT, Brands AM (2012) Cognitive impairment in multiple sclerosis. Minerva Med 103: 73-96.

36. Portaccio E, Stromillo ML, Goretti B, Zipoli V, Siracusa G, et al. (2009) Neuropsychological and MRI measures predict short-term evolution in benign multiple sclerosis. Neurology 73: 498-503. 